\title{
NON-SQUARE QUANTUM WELL GROWTH FOR REDUCED THRESHOLD CURRENT IN TENSILELY STRAINED LASERS OPERATING AT $1.52 \mu \mathrm{m}$
}

\author{
K.A. Kaduki \\ Department of Physics, University of Nairobi, \\ P. O. Box 30197, Nairobi, KENYA
}

\begin{abstract}
This paper presents calculations demonstrating that non-square quantum well growth (well shaping) can result in reduced threshold current for tensilely strained quantum well bipolar diode lasers operating at $1.52 \mu \mathrm{mm}$. Calculations of subband structure, optical matrix elements and laser gain are performed for arbitrarily shaped quantum wells based on a 4-band (electron/heavyhole/light-hole/split off-hole) Hamiltonian. For long wavelength $\left(1.3 \mu \mathrm{m} m\right.$ to $\left.1.55 \mu_{\mathrm{m}}\right)$ lasers, Auger recombination dominates the threshold current. Compared to a $1.52 \mathrm{~mm}$ optimal square well just below critical thickness, an InGaAs-InGaAsP (on InP) well incorporating potential 'spikes' and having the same wavelength can be much wider. The wider well, possible with well shaping, results in a lower value for three-dimensional (3D) carrier density at a given value of modal gain. For low loss lasers, this implies a reduction in Auger (and hence total) threshold current to a value below the best obtainable in a laser based on a square quantum well.
\end{abstract}

\section{INTRODUCTION}

Long wavelength $(1.3 \mu \mathrm{m}$ to $1.55 \mu \mathrm{m})$ strained quantum well $(\mathrm{QW})$ laser diodes have been widely studied since they are key components for optical communication systems. Incorporating strain into the active region of the laser gives such benefits as reduced threshold current, high modulation speed, smaller linewidth enhancement factor and reduced temperature sensitivity. The material of choice for lasers operating at $\sim 1.55 \mu \mathrm{m}$ is InGaAsInGaAsP (on InP) for which the benefits of strain has been proven. It has been reported that tensilely strained quantum wells perform as well as if not better than compressively strained quantum wells [1]. Benefits derived from use of tensile strain have been attributed to reduced level of spontaneous emission and an enhancement of the transition matrix $[2,3]$. On the other hand, the technique of non-square quantum well growth (well shaping) has mainly been applied to the enhancement of photo-detectors [4], optical modulators [5, 6], intersubband (unipolar) lasers [7] and non-linear devices [8, 9]. In recent calculations [10], it was shown that combining strain with an appropriately shaped compressively strained stepped alloy quantum well can lead to reduced threshold current in bipolar laser diodes. This work extends the technique of well shaping to include lasers incorporating tensilely strained quantum wells.
Tensilely strained InGaAs-InGaAsP square quantum well (SQW) lasers having a fixed emission wavelength show a reduction in threshold current as the well width is increased within the limits of critical layer thickness [1]. Silver and O'Reilly [11] have suggested that the increased well width plays a part in reducing the three-dimensional (3D) carrier density in the active region of these lasers, thereby reducing the Auger recombination rate. The minimum threshold current is achieved just before the well width reaches critical thickness. It has now been firmly established that Auger recombination is responsible for up to $80 \%$ of the threshold current in long-wavelength (1.3 to $1.55 \mu \mathrm{m}$ ) InGaAs-InGaAsP lasers operating at room temperature $[12,13,14]$.

Incorporation of one or two narrow potential spikes into a square quantum well while at the same time keeping the well width fixed results in an increase in the fundamental electron-hole transition energy. On the other hand, increasing the well width of such a 'spiked' quantum well has the counter effect of reducing the transition energy. For a fixed transition energy therefore, a spiked quantum well will be much wider than a conventional square quantum well. In this paper, it is shown computationally that the wider spiked quantum wells have reduced threedimensional (3D) carrier density at threshold when used in the active region of bipolar diode lasers. This leads to a reduction in the dominant Auger current component. 
The paper is organised as follows. First the theoretical model used in the calculation of the quantum well energy bandstructures, optical gain and radiative current density is described. The results are then presented and discussed, followed by the conclusions of the study.

\section{THEORETICALMODEL}

\section{Band structure Calculations for a Strained Quantum Well}

Consider a quantum well of width $L_{z}$ grown along $z$ with a biaxial strain. For a direct bandgap semiconductor, the bandstructure near the zone centre can be described by the k.p method [15]. In this work, an 8-band effective mass Hamiltonian based on the $\mathbf{k} \times \mathbf{p}$ method is used to describe the coupled QW electron (EL), heavy-hole (HH), light-hole (LH) and split-off-hole (SO) states. By use of a unitary transformation, this $8 \times 8$ Hamiltonian is block diagonalized (decoupled) into two 4'4 Hamiltonians whose wavefunctions are given by [16]

$$
\Psi_{n k_{/ /}}^{\sigma}(\mathbf{r})=\frac{1}{\sqrt{A}} \sum_{v} F_{n}^{(v)}\left(k_{/ /}, z\right) \mathrm{e}^{i \mathbf{k}_{\|} \cdot \vec{\rho}}\left|u_{v}^{\prime}\right\rangle
$$

where $\mathrm{s}=+\mathrm{ve}$ for the upper-block and $\mathrm{s}=-\mathrm{ve}$ for the lowerblock. The summations are over $n=1,2,3,4$ and $n=5,6,7,8$ respectively with $\left\{F_{n}^{(v)}\left(k_{/ /}, z\right)\right\}$ being the associated envelope functions $\mathbf{k}_{\|}\left(=k_{x} \hat{\mathbf{x}}+k_{y} \hat{\mathbf{y}}\right)$ is the in-plane wavevector with $\hat{\mathbf{x}}$ and $\hat{\mathbf{y}}$ unit vectors; $A$ the normalisation area of the quantum well; $\left\{\left|v^{\prime}\right\rangle\right\}$ the transformed zone centre Bloch functions while $\vec{\rho}=x \hat{\mathbf{x}}+y \hat{\mathbf{y}}$. The decoupled Hamiltonians are [16]

$$
\begin{aligned}
& \mathbf{H}^{ \pm}\left(k_{/ /}, \hat{k}_{z}\right)= \\
& \left(\begin{array}{cccc}
f+S_{h y d}^{C} & \mp g & \pm \frac{g}{\sqrt{3}}+h & \pm \sqrt{\frac{2}{3}} g+\frac{h^{*}}{\sqrt{2}} \\
\mp g & a_{+}+S_{h y d}^{V}+S & c \pm i K & \sqrt{2} c \mp i \frac{K}{\sqrt{2}} \\
\pm \frac{g}{\sqrt{3}}+h^{*} & c \mp i K^{\dagger} & a_{-}+i J+S_{h y d}^{V}-S & e \pm i \sqrt{\frac{3}{2}} L+\sqrt{2} S \\
\pm \sqrt{\frac{2}{3}} g+\frac{h}{\sqrt{2}} & \sqrt{2} c \pm i \frac{K^{\dagger}}{\sqrt{2}} & e \mp i \sqrt{\frac{3}{2}} L^{\dagger}+\sqrt{2} S & d \mp i J+S_{h y d}^{V}
\end{array}\right)
\end{aligned}
$$

with

$$
\begin{aligned}
& f=E_{C 0}+\left(\frac{\hbar^{2}}{2 m_{o}}\right)\left[(2 F+1) k_{/ /}^{2}+\hat{k}_{z}(2 F+1) \hat{k}_{z}\right] \\
& a_{+}=-E_{V O}-\left(\frac{\hbar^{2}}{2 m_{o}}\right)\left[\left(\gamma_{1}+\gamma_{2}\right) k_{/ /}^{2}+\hat{k}_{z}\left(\gamma_{1}-2 \gamma_{2}\right) \hat{k}_{z}\right. \text {. } \\
& a_{-}=-E_{V O}-\left(\frac{\hbar^{2}}{2 m_{o}}\right)\left[\left(\gamma_{1}-\gamma_{2}\right) k_{/ /}^{2}+\hat{k}_{z}\left(\gamma_{1}+2 \gamma_{2}\right) \hat{k}_{z}\right] \\
& d=-E_{V O}-\Delta_{O}-\left(\frac{\hbar^{2}}{2 m_{o}}\right)\left[\gamma_{1} k_{/ /}^{2}+\hat{k}_{z} \gamma_{1} \hat{k}_{z}\right] \\
& b=\left(\frac{\hbar^{2}}{2 m_{o}}\right) 2 \sqrt{3} k_{/ /}\left\{\gamma_{3}, \hat{k}_{z}\right\}
\end{aligned}
$$$$
c=\left(\frac{\hbar^{2}}{2 m_{o}}\right) \sqrt{3} \gamma^{\prime} k_{\mu /}^{2} ; \gamma^{\prime}= \begin{cases}\frac{1}{2}\left(\gamma_{2}+\gamma_{3}\right) & \text { axial approximation } \\ \gamma_{2} & \text { along }\langle 100\rangle \\ \gamma_{3} & \text { along }\langle 110\rangle\end{cases}
$$$$
e=\left(\frac{\hbar^{2}}{2 m_{o}}\right) \sqrt{2}\left[2 \hat{k}_{z} \gamma_{2} \hat{k}_{z}-\gamma_{2} k_{/ /}^{2}\right]
$$$$
g=\frac{1}{\sqrt{2}} P k_{/ \prime}
$$$$
h=\sqrt{\frac{2}{3}} i\left\{P, \hat{k}_{z}\right\}
$$$$
J=\left(\frac{\hbar^{2}}{2 m_{o}}\right) k_{/ /}\left[\hat{k}_{z}(\sigma-\delta-\pi)-(\sigma-\delta-\pi) \hat{k}_{z}\right]
$$$$
K=\left(\frac{\hbar^{2}}{2 m_{o}}\right) \sqrt{3} k_{/ /}\left[(\sigma-\delta) \hat{k}_{z}+\hat{k}_{z}(\pi)\right]
$$$$
L=\left(\begin{array}{c}
\frac{\hbar^{2}}{2 m_{o}} \\
)
\end{array}\right) \sqrt{3} k_{/ /}\left\{\left[\frac{1}{3}(\sigma-\delta)+\frac{2}{3} \pi\right] \hat{k}_{z}+\hat{k}_{z}\left[\frac{2}{3}(\sigma-\delta)+\frac{1}{3} \pi\right]\right\}
$$

where

$$
\begin{aligned}
\delta & =\frac{1}{9}\left(1+\gamma_{1}+\gamma_{2}-3 \gamma_{3}\right) \\
\sigma & =\frac{1}{2}\left(\gamma_{3}+\gamma_{2}\right)-\frac{1}{2} \delta \\
\pi & =\frac{1}{2}\left(\gamma_{3}-\gamma_{2}\right)+\frac{3}{2} \delta . \\
k_{/ /}^{2} & =k_{x}^{2}+k_{y}^{2} ; \quad \hat{k}_{z}=-i \frac{\mathrm{d}}{\mathrm{d} z}
\end{aligned}
$$

$E_{C O}\left(E_{V O}\right)$ is the conduction (valence) band edge at the $\mathrm{G}$ -point, $\mathrm{D}_{O}$ the spin orbit splitting while $P$ and $F$ are, respectively, the first and second order Kane parameters for the conduction band. $F$ is related to the experimentally determined conduction band effective mass $m_{c}^{*}$ by [17]

$$
\frac{1}{m_{c}^{*}}=(2 F+1)+\frac{E_{P}}{3}\left[\frac{2}{E_{g}}+\frac{1}{E_{g}+\Delta_{O}}\right]
$$

where $E_{g}$ is the fundamental gap and $E_{P}=\left(2 m_{o} P^{2}\right) / \hbar^{2}$. $S_{\text {hyd }}^{C}$ and $S_{\text {hyd }}^{V}$ are the strain induced hydrostatic shifts of the conduction and valence band edges respectively and $S$ the shear strain shift. The parameters $\gamma_{1}, \gamma_{2}$ and $\gamma_{3}$ are related to the Luttinger gamma parameters $\left(\gamma_{1}^{L}, \gamma_{2}^{L}\right.$ and $\gamma_{3}^{L}$ ) via the relations 
$\gamma_{1}^{L}=\gamma_{1}+E_{P} /\left(3 E_{g}\right)$

$\gamma_{2}^{L}=\gamma_{2}+E_{P} /\left(6 E_{g}\right)$

$\gamma_{3}^{L}=\gamma_{2}+E_{P} /\left(6 E_{g}\right)$

The envelope functions satisfy

$\sum_{v^{\prime}=1}^{4}\left[H_{v v^{\prime}}^{+}\left(k_{/ /},-i \frac{\mathrm{d}}{\mathrm{d} z}\right)\right] F_{n}^{\left(v^{\prime}\right)}\left(k_{/ /}, z\right)=E_{n}\left(k_{/ /}\right) F_{n}^{(v)}\left(k_{/ /}, z\right)$

(7)

and

$\sum_{v^{\prime}=1}^{4}\left[H_{v v^{\prime}}^{-}\left(k_{/ /},-i \frac{\mathrm{d}}{\mathrm{d} z}\right)\right] F_{n}^{\left(v^{\prime}+4\right)}\left(k_{/ /}, z\right)=E_{n}\left(k_{/ /}\right) F_{n}^{(v+4)}\left(k_{/ /}, z\right)$

for $\mathrm{n}=1,2,3,4$.

$H_{v v^{\prime}}^{ \pm}$in Equation 8 are the components of the upper (+) and lower (-) blocks of the multi-band effective mass Hamiltonian [Eq.2] respectively. Equations (7) and (8) are solved by basis function expansion in terms of solutions, obtained by an accurate shooting method, of the corresponding single band problems. Details of the calculation are given in [16].

\section{Optical Properties of Strained QW Lasers}

Using the output from the bandstructure calculation, the optical gain at photon energy $\hbar \omega$ is calculated according to the expression [18]

$$
\begin{aligned}
g(\hbar \omega)= & \frac{e^{2} \pi}{n_{w} c \varepsilon_{o} m_{o}^{2} \omega L_{z}} \sum_{\sigma \sigma^{\prime}} \sum_{n m} \int\left|\lambda \cdot \mathbf{M}_{n m}^{\sigma \sigma^{\prime}}\left(k_{/ /}\right)\right|^{2} \times \\
& \frac{\left[f_{n}^{C}\left(k_{/ /}\right)-f_{m}^{V}\left(k_{/ /}\right)\right]\left(\hbar / \pi \tau_{i n}\right)}{\left(E_{n}^{C}\left(k_{/ /}\right)-E_{m}^{V}\left(k_{/ /}\right)-\hbar \omega\right)^{2}+\left(\hbar / \tau_{i n}\right)^{2}} \frac{k_{/ /} d k_{/ /}}{2 \pi}
\end{aligned}
$$

(9)

where $n_{w}$ is the refractive index, $m_{o}$ is the free electron mass, $\mathrm{e}_{o}$ is the free space dielectric constant, $c$ is the velocity of light, 1 the polarisation vector of the optical field and $e$ the magnitude of the electronic charge. $f_{n}^{C}\left(k_{/ /}\right)$ and $f_{m}^{V}\left(k_{/ /}\right)$are the electron occupation probabilities for the conduction and valence bands respectively with $\tau_{\text {in }}$ the intra-band relaxation time, assumed here to be $10^{-13} \mathrm{~s}$. $\hbar=h / 2 \pi$ where $h$ is Plank's constant. $E_{n}^{C}\left(k_{/ /}\right)$and

$E_{m}^{V}\left(k_{/ /}\right)$are the conduction and valence subband energies respectively and $\mathbf{M}_{n m}^{\sigma \sigma^{\prime}}\left(k_{/ /}\right)$the momentum matrix elements between conduction subbands $n$ and valence subbands $m$. The momentum matrix elements are defined in [10].

The rate of radiative transitions due to the spontaneous emission process is [18]

$R_{s p}=\int r_{s p}(\hbar \omega) d(\hbar \omega)$

where

$$
\begin{gathered}
r_{s p}(\hbar \omega)=\frac{e^{2} n_{w} \omega}{\pi \hbar c^{3} \varepsilon_{o} m_{o}^{2} L_{z}} \sum_{\sigma \sigma^{\prime}} \sum_{n m} \int\left|M_{n m}^{\sigma \sigma^{\prime}}\left(k_{/ /}\right)\right|_{s p}^{2} \times \\
\frac{f_{n}^{C}\left(k_{/ /}\right)\left[1-f_{m}^{V}\left(k_{/ /}\right)\right]}{\left(E_{n}^{C}\left(k_{/ /}\right)-E_{m}^{V}\left(k_{/ /}\right)-\hbar \omega\right)^{2}+\left(\hbar / \tau_{i n}\right)^{2}} \frac{k_{/ /} d k_{/ /}}{2 \pi}
\end{gathered}
$$

with $\mathbf{M}_{n m}^{\sigma \sigma^{\prime}}\left(k_{/ /}\right)_{s p}$ being the spontaneous emission optical matrix elements [10]. The radiative component of the current density is then evaluated using

$J^{R}=e L_{z} R_{s p}$

The Auger component of the current density $\left(J^{A}\right)$ is given by [19]

$$
J^{A}=e L_{z} C\left(n^{3 D}\right)^{3}
$$

where $C$ is the three-dimensional (3D) Auger coefficient, the $3 \mathrm{D}$ carrier density and $L_{z}$ is the well width. In lasers, the threshold condition occurs when the peak gain equals the total losses in the optical cavity. The total current density is the sum of $J^{R}$ and $J^{A}$. 


\section{NUMERICAL RESULTS AND DISCUSSION}

This section presents results that demonstrate the improvement of threshold current performance in bipolar diode lasers through well shaping. Laser characteristics of tensilely strained InGaAs/InGaAsP (on InP) square and shaped quantum wells with an emission wavelength of $1.52 \mathrm{~mm}$ are compared. At this wavelength, Auger recombination is the dominant carrier loss mechanism at room temperature, contributing up to $80 \%$ of the total threshold current $[12,13,14]$. In this work, only the influence of stepped alloy growth on laser performance is examined. Well shaping techniques such as delta-doping [20]and inter-diffusion [21] are not considered.

\section{Energy Bandstructure}

The energy bandstructure was calculated following the model outlined in the previous section. A $175 \AA$ tensilely strained $\mathrm{In}_{0.36} \mathrm{Ga}_{0.64}$ As square quantum well (SQW) with $\mathrm{In}_{0.91} \mathrm{Ga}_{0.09} \mathrm{As}_{0.2} \mathrm{P}_{0.8}$ barriers is taken as the reference system. This SQW has transition energy of $815 \mathrm{meV}$, equivalent to an emission wavelength of $1.52 \mathrm{~mm}$. The non-square quantum well considered here is designed to be much wider than the SQW yet within the critical layer thickness limit. The critical thickness is calculated using the expression proposed by Matthews and Blakeslee [22].

Figure 1 illustrates the conduction and light hole valence band profiles for the square and non-square quantum wells. The heavy-hole and split-off hole potential profiles are similar to the light-hole profile shown. The non-square quantum well is referred to as 'spiked quantum well' (SpQW) for obvious reasons. The heavy-hole $(\mathrm{HH})$ and split-offhole (SO) well profiles are similar to the light-hole profile. The SpQW has a wider well than the SQW, i.e. $\mathrm{L}_{2}>\mathrm{L}_{1}$. Material compositions of the specific structures considered in this paper are given in Table 1.

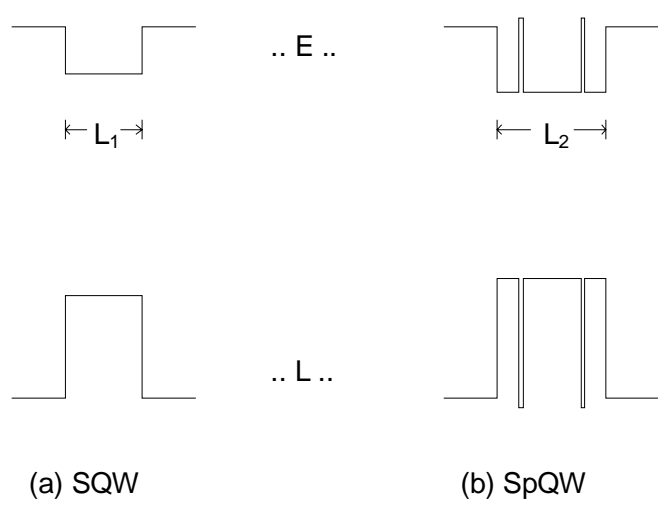

Figure 1: Schematic diagram of the electron (EL) and light-hole (LH) band edge profiles for (a) a square quantum well (SQW) and (b) a 'spiked' quantum well (SpQW).
Table 1. Material parameters for the spiked quantum well (SpQW) and the two square quantum wells (SQWs).

\begin{tabular}{|l|c|c|c|c|}
\hline & $\mathrm{A}$ & $\mathrm{B}$ & $\mathrm{D}$ & $\mathrm{E}$ \\
\hline$E_{g}(\mathrm{eV})$ & 0.936 & 0.888 & 1.274 & 1.201 \\
\hline$\Delta E_{c}^{D}(\mathrm{eV})$ & -0.115 & -0.139 & - & - \\
\hline$\Delta E_{c}^{E}(\mathrm{eV})$ & -0.086 & -0.112 & - & - \\
\hline$m_{c}^{*}$ & 0.049 & 0.047 & 0.076 & 0.073 \\
\hline$\gamma_{1}^{L}$ & 9.27 & 9.59 & 5.38 & 5.73 \\
\hline$\gamma_{2}^{L}$ & 3.45 & 3.6 & 1.76 & 1.89 \\
\hline$\gamma_{3}^{L}$ & 4.09 & 4.25 & 2.24 & 2.41 \\
\hline$S_{h y d}^{c}(\mathrm{meV})$ & -81.4 & -61.2 & 0 & 0 \\
\hline$S_{h y d}^{v}(\mathrm{meV})$ & 14.9 & 11 & 0 & 0 \\
\hline$S(m e V)$ & -45.3 & -34.6 & 0 & 0 \\
\hline$\Delta_{o}(\mathrm{eV})$ & 0.322 & 0.32 & 0.149 & 0.167 \\
\hline$E_{P}(\mathrm{eV})$ & 24.3 & 24.3 & 24.3 & 24.3 \\
\hline
\end{tabular}

The barrier material in the SpQW is unstrained $1.20 \mathrm{eV}$ $\mathrm{In}_{0.91} \mathrm{Ga}_{0.09} \mathrm{As}_{0.2} \mathrm{P}_{0.8}$. The structure has two unstrained 1.27 $\mathrm{eV}, 12 \AA$ wide $\mathrm{In}_{0.95} \mathrm{Ga}_{0.05} \mathrm{As}_{0.1} \mathrm{P}_{0.9}$ spikes separated by $152 \AA$ incorporated symmetrically into the $254 \AA$ wide $\operatorname{In}_{0.4} \mathrm{Ga}_{0.6} \mathrm{As}$ well region. The composition and separation of the spikes are chosen to keep the emission wavelength fixed at 815 $\mathrm{meV}$ while at the same time maintaining as large a separation as possible between the ground and first excited state electron, heavy-hole, light-hole and split-off hole subbands. The use of the potential spikes makes possible the design of quantum wells that are much wider than is possible with conventional square wells at a fixed emission wavelength, as determined by critical thickness constraints.

The room temperature material parameters for the two structures have been interpolated from the data of constituent binary alloys given in [23] and are shown in Table 1. Bowing parameters for the energy gaps are taken from [24] while the unstrained valence band offset for the InGaAs-InGaAsP interface is calculated following Weihofen [25] $\Delta E_{c}^{D}$ and $\Delta E_{c}^{E}$ are the strained conduction band offsets with respect to barrier materials $\mathrm{D}$ $\left(\mathrm{In}_{0.95} \mathrm{Ga}_{0.05} \mathrm{As}_{0.1} \mathrm{P}_{0.9}\right)$ and $\mathrm{E} \quad\left(\mathrm{In}_{0.91} \mathrm{Ga}_{0.09} \mathrm{As}_{0.2} \mathrm{P}_{0.8}\right)$ respectively.

Material $\mathrm{A}$ is $\mathrm{In}_{0.36} \mathrm{Ga}_{0.64} \mathrm{As}, \mathrm{B}$ is $\mathrm{In}_{0.4} \mathrm{Ga}_{0.6} \mathrm{As}$, and $\mathrm{D}$ is $\mathrm{In}_{0.95} \mathrm{Ga}_{0.05} \mathrm{As}_{0.1} \mathrm{P}_{0.9}$ while $\mathrm{E}$ is $\mathrm{In}_{0.91} \mathrm{Ga}_{0.09} \mathrm{As}_{0.2} \mathrm{P}_{0.8}$. The well regions of the $175 \AA$ and $150 \AA$ wide structures are formed from materials $A$ and $B$ respectively, while the $254 \AA \mathrm{ApQW}$ has a material $\mathrm{B}$ well region and material $\mathrm{D}$ spikes. The barrier layers for all the structures are $\mathrm{In}_{0.91} \mathrm{Ga}_{0.09} \mathrm{As}_{0.2} \mathrm{P}_{0.8}$ 
(material E). The parameters are defined in the text.

The $175 \AA$ SQW is just below the calculated critical thickness and is theoretically the widest square quantum well attainable at $1.52 \mathrm{~mm}$. Based on the trends reported in $[1,11]$, namely that the threshold current falls with increasing well width and strain, it is expected that the $175 \AA$ SQW will have the lowest threshold current available in an SQW in this material system at $1.52 \mathrm{~mm}$. A non-optimal, $150 \AA$ wide, $1.52 \mathrm{~mm} \mathrm{SQW}$ of the same material composition $\left(\mathrm{In}_{0.4} \mathrm{Ga}_{0.6} \mathrm{As}\right)$ as the strained region of the SpQW has also been included in the study for purposes of comparison.

Figures 2 and 3 show the calculated valence band structure for the $254 \AA \mathrm{SpQW}$ (dashed lines in each case) compared with the valence bandstructure of the $150 \AA$ SQW and the $175 \AA$ SQW (solid lines in each case). The zero of energy is taken to coincide with the zone centre energy of the first hole subband. From these figures one can see that for the SpQW, separation between the first two hole subbands is comparable to that of the two SQWs. Also, the in-plane mass (as reflected by the subband curvature) of the ground state subband in the SpQW is seen to be nearly equal to that in the $150 \AA \mathrm{SQW}$, but significantly heavier than that in the $175 \AA$ SQW. The gain and radiative current density characteristics based on the bandstructures of Figures 2 and 3 are presented next.

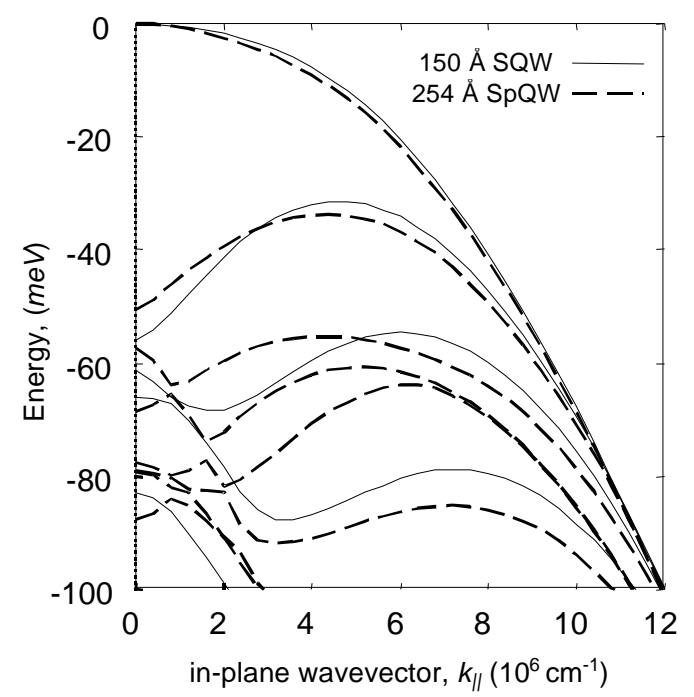

Figure 2: Variation of subband energy with in-plane wavevector $(\mathrm{k}$ ) for the $150 \AA \mathrm{SQW}$ (solid lines) and the $254 \AA \mathrm{SpQW}$ (dashed lines) described in Figure 1 and Table 2. The ordering of the three highest lying subbands is LH1-HH1-LH2 for the SQW and LH1LH2-HH1 for the SpQW.

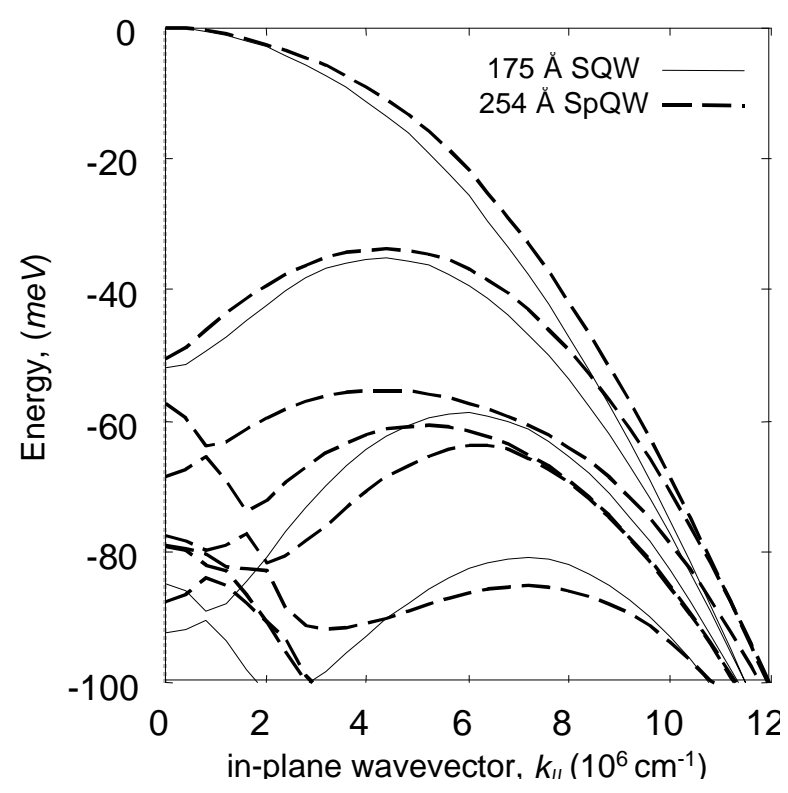

Figure 3: Variation of subband energy with in-plane wavevector $(\mathrm{k}$ ) for the $175 \AA \mathrm{SQW}$ (solid lines) and the $254 \AA \mathrm{SpQW}$ (dashed lines) described in Figure 1 and Table 2. The ordering of the three highest lying subbands is LH1-LH2-HH1 for the both SQW and the SpQW.

\section{Optical Gain and Radiative Current Density Characteristics}

The optical gain and radiative density characteristics were calculated using Equations 9 and 10 using energy dispersions and optical matrix elements from the bandstructure calculations. Since the quantum wells have different widths, the modal gain $(\mathrm{Gg})$, rather than the material gain $(g)$, is plotted to facilitate comparison. For a single step index separate confinement heterostructure (SCH) laser with a single quantum well of width $L_{z}$ and an optical cavity of width $d$, the TM optical confinement factor $\left(\mathrm{G}_{T M}\right)$ is given by

$\Gamma_{T M}=\left(\frac{n_{b}}{n_{w}}\right)^{2} \frac{L_{z}}{d}$

Where $n_{b}$ is the barrier refractive index. Only the TM mode is considered since TE transitions are suppressed in tensilely strained structures [3]. The refractive indices have been calculated using the modified single effective oscillator model [i], ii] ] and are listed in Table 2 along with the optical confinement factor calculated for a laser having an optical cavity width of $0.45 \mathrm{~mm}$. The barrier refractive index is 3.222 in all cases. 
Table 2. Calculated refractive index and optical confinement factor for the $150 \AA$ and $175 \AA$ square quantum wells (SQWs) and the $254 \AA$ spiked quantum well (SpQW).

\begin{tabular}{|c|c|c|}
\hline Structure & $\begin{array}{c}\text { Refractive } \\
\text { Index }\left(n_{w}\right)\end{array}$ & $\begin{array}{c}\text { Optical } \\
\text { Confinement } \\
\text { Factor }(\Gamma)\end{array}$ \\
\hline $150 \AA$ SQW & 3.481 & 0.0285 \\
\hline $175 \AA$ SQW & 3.466 & 0.0336 \\
\hline $254 \AA$ SpQW & 3.452 & 0.0491 \\
\hline
\end{tabular}

Figure 4 shows the variation of modal TM gain with radiative current density (RCD) for the three structures. The SpQW has the highest radiative current density for all values of modal gain. At low modal gain, the $150 \AA$ SQW has marginally lower radiative current density performance than the $175 \AA \mathrm{SQW}$. This situation is reversed from about $15 \mathrm{~cm}^{-1}$, when the $175 \AA$ structure gives significantly lower $\mathrm{RCD}$ values with increasing modal gain. These results can be explained using the energy dispersions of Figures 2 and 3. Close spacing of the subbands leads to significant contribution of the excited states to the spontaneous emission and hence the radiative current. The $175 \AA \mathrm{SQW}$ has the largest subband separations and therefore has the best characteristics overall. The close spacing of the excited state subbands of the SpQW is responsible for its comparatively poor performance.

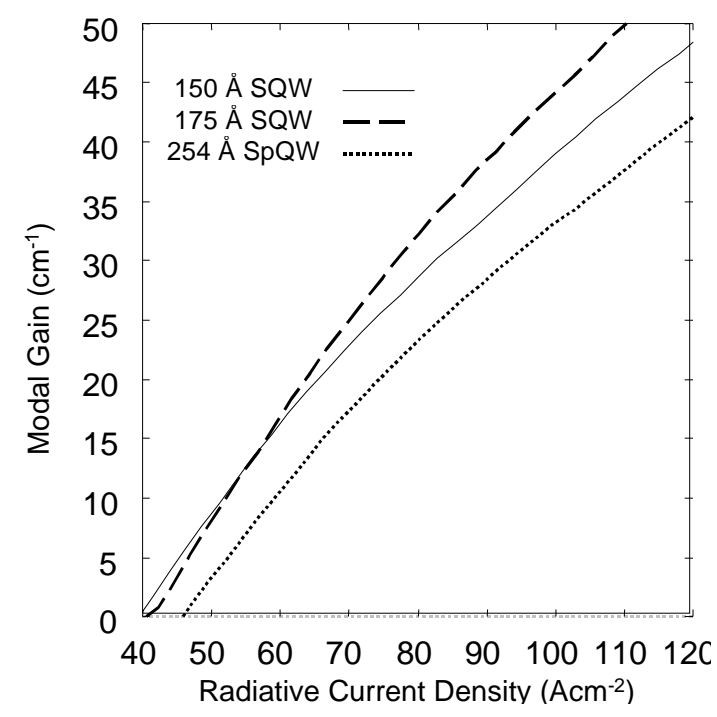

Figure 4: Modal TM gain as a function of radiative current density (RCD) for the $150 \AA$ and $175 \AA$ SQWs and the $254 \AA$ SpQW for a separate confinement heterostructure $(\mathrm{SCH})$ laser with an optical cavity width of $0.45 \mathrm{~mm}$.
From these results, it is clear that the shaped quantum well structure presented in this paper is not expected to help reduce the radiative component of the threshold current compared to the optimised SQW. The radiative current component is however expected to be small ( $\sim 20 \%$ of total current) compared to the Auger component $[12,13,14]$. It is now shown that the SpQW has lower Auger current density than the optimal SQW in low loss laser cavities, leading to lower total threshold current density.

In their study of tensilely strained InGaAs-InGaAsP laser structures, Jones et al.[28] show that quantum well structures having well widths greater than $\sim 150 \AA$ exhibit bulk-like behaviour. Since the three structures in this study satisfy this criterion, the calculated gain has been plotted against the three-dimensional carrier density as opposed to the usual two-dimensional carrier density. The variation of modal TM gain with the 3D carrier density is shown in Figure 5 for the $150 \AA$ SQW (solid line), the $175 \AA$ SQW (dashed line) and the $254 \AA$ SpQW (dotted line). It is seen that the $254 \AA \mathrm{SpQW}$ has the highest values of modal gain at any given value of $3 \mathrm{D}$ carrier density compared with the two SQWs.

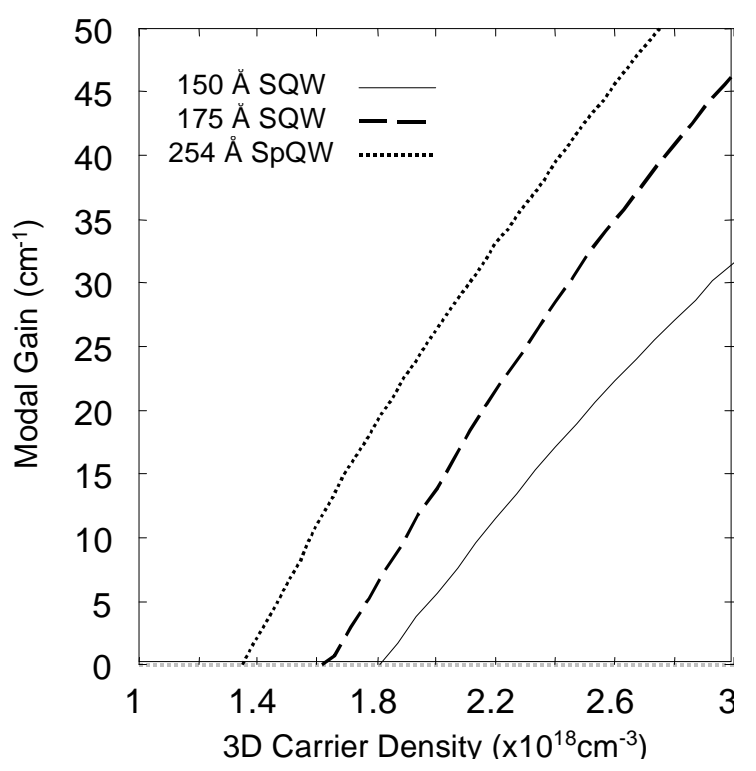

Figure 5: Plots of the modal TM gain as a function of the $3 \mathrm{D}$ carrier density for the $150 \AA$ and $175 \AA$ SQWs and the $254 \AA$ SpQW for a separate confinement heterostructure $(\mathrm{SCH})$ laser with an optical cavity width of $0.45 \mathrm{~mm}$.

While the radiative current density (RCD) at threshold can be determined directly from the gain/RCD characteristics (Figure 4), evaluation of the dominant Auger current density requires knowledge of the Auger coefficient (C). The Auger current is calculated using Equation 12. Values 
of C vary from one material system to another and depend on the type of recombination process which is dominant, i.e., whether direct or phonon assisted. A number of authors have published values of Auger coefficients for InGaAs and InGaAsP quantum well structures with $1.55 \mathrm{~mm}$ emission wavelength $[29,30,31,32]$ However, most of the work reported in these references focuses on quantum well structures under compressive strain. Relatively little systematic work has been done for tensilely strained structures [12]. There clearly is a need to characterise experimentally the Auger coefficients of quantum well structures for a wider range of material compositions and systems. Given that the structures studied in this work are under tensile strain, and in the light of the shortcomings just presented, only a comparative assessment of their performance as lasers is presented.

\section{Evaluation}

It has been shown both theoretically and experimentally that the value of Auger coefficient $(C)$, at worst, remains constant as the well width is increased while keeping the emission wavelength constant [29,30,31]. In the calculations that follow therefore, a value of is assumed for the two SQWs and the SpQW. The Auger component of the threshold current is calculated using the data of Figure 5 and Equation 12 for the different values of modal gain. The total current is obtained by adding the Auger component to the radiative component of Figure 4. The result is shown in Figure 6.

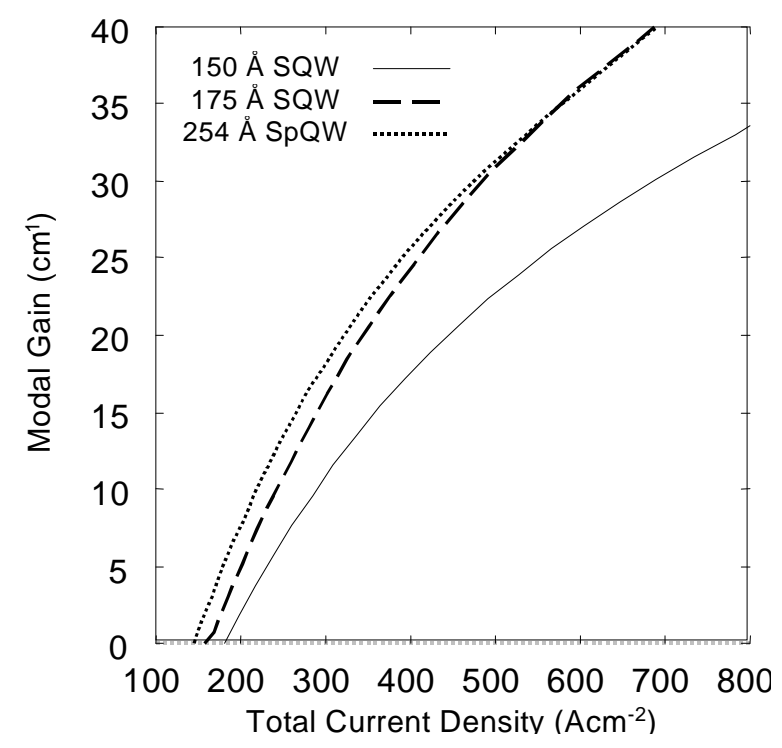

Figure 6: Variation of modal TM gains with total current density for the $150 \AA$ and $175 \AA$ SQWs and the $254 \AA$ SpQW for a separate confinement heterostructure $(\mathrm{SCH})$ laser with an optical cavity width of $0.45 \mathrm{~mm}$.
Of the three structures, the $150 \AA \mathrm{SQW}$ has the highest values of total current density for all values of modal gain. The $254 \AA$ SpQW has the lowest values of total current density for lasers with cavity losses between $0 \mathrm{~cm}^{-1}$ and $\sim 30 \mathrm{~cm}^{-1}$ while, beyond $\sim 30 \mathrm{~cm}^{-1}$, the $175 \AA$ has the lowest total current density. A laser incorporating an SpQW will therefore have the best threshold current performance at $1.52 \mathrm{~mm}$ in the low loss regime. Table 3 shows the calculated total gain for the three structures at modal gain values of 5 $\mathrm{cm}^{-1}, 10 \mathrm{~cm}^{-1}$ and $20 \mathrm{~cm}^{-1}$.

Table 3: Calculated values of total threshold current density at modal threshold gain values of $5 \mathrm{~cm}^{-1}, 10 \mathrm{~cm}^{-1}$ and $20 \mathrm{~cm}^{-1}$ for the two SQWs and for the SpQW.

\begin{tabular}{|c|c|c|c|}
\hline & \multicolumn{2}{|c|}{$\begin{array}{c}\text { TOTAL THRESHOLD CURRENT } \\
\text { DENSITY }\left(\mathrm{Acm}^{-2}\right)\end{array}$} \\
\hline & $g_{\text {th }}^{m}=5 \mathrm{~cm}^{-1}$ & $g_{\text {th }}^{m}=10 \mathrm{~cm}^{-1}$ & $g_{\text {th }}^{m}=20 \mathrm{~cm}^{-1}$ \\
\hline $150 \AA$ & 238 & 285 & 457 \\
$\mathrm{SQW}$ & & & \\
\hline $175 \AA$ & 202 & 240 & 351 \\
$\mathrm{SQW}$ & & & \\
\hline $254 \AA$ & 180 & 218 & 321 \\
$\mathrm{SpQW}$ & & & \\
\hline
\end{tabular}

Compared to the $175 \AA \mathrm{SQW}$, the $254 \AA \mathrm{SpQW}$ design gives reductions in total current density of $10.9 \%$ (at $5 \mathrm{~cm}^{-1}$ ), $9.2 \%\left(\right.$ at $10 \mathrm{~cm}^{-1}$ ) and $8.5 \%$ (at $20 \mathrm{~cm}^{-1}$ ). It is worth noting that the results shown in Figure 6 and Table 3 represent the worst-case scenario for the wide, $254 \AA$, SpQW as its Auger coefficient $C$ is expected to be smaller than that of the narrower SQWs. Using a smaller value of $C$ for the SpQW should result in a larger reduction of the total current density for the SpQW.

\section{SUMMARY AND CONCLUSION}

The electronic and optical properties of quantum wells of arbitrary shape have been calculated using a multi-band effective mass model that explicitly incorporates coupling between the lowest lying conduction band and the highest lying valence bands. It has been shown that well shaping by stepped alloy growth can be beneficial for reducing the threshold current in bipolar diode lasers operating at a wavelength of $1.52 \mathrm{~mm}$.

At $1.52 \mathrm{~mm}$, Auger current $\left(J^{A}\right)$ dominates the laser threshold current and since $J^{A} \sim L_{z} C\left(n^{3 D}\right)^{3}$, a reduction in the $3 \mathrm{D}$ carrier density $n^{3 D}$ will result in lower threshold current laser operation. For tensilely strained InGaAsInGaAsP structures, shaping of the quantum wells using potential 'spikes' produces a significantly wider well than an optimal square quantum well of the same emission 
wavelength and barrier composition. This leads to a reduced 3D carrier density, and so a reduction of the Auger current for low loss bipolar diode lasers. This prediction is arrived at by assuming that the 3D Auger coefficient is independent of well width for relatively wide wells $[29,30]$. The specific $254 \AA$ spiked quantum well (SpQW) considered has a smaller value of $3 \mathrm{D}$ carrier density at all values of modal gain than an optimal $175 \AA$ square quantum well (SQW). On the other hand, the modal gain/radiative current density characteristics are not improved for the SpQW, leading to a larger value of radiative threshold current density than for the SQW laser. However, since Auger current dominates, the increased radiative current component of the SpQW is more than offset by the reduced Auger component, leading to reduced total threshold current density for a low loss, bipolar diode laser.

\section{REFERENCES}

[1] Thijs P.J.A., Tiemeijer L.F., Binsma J.J.M. and van Dongen T., IEEE J. Quantum Electron., 30, p.477 499, 1994.

[2] O'Reilly E.P., Jones G., Ghiti A. and Adams A.R., Electron. Lett., 27, p1417-1419, 1991.

[3] Jones G and O'Reilly E.P., IEEE J. Quantum Electron., 29, p.1344-1354, 1993.

[4] Trzeciakowski W. and McCombe, B.D, Appl. Phys. Lett., 55, p.891-893, 1989.

[5] Guettler T., Krebs O., Dias I.L., Harmand J.C., Devaux F. and Voisin P., Semicond. Sci. Technol., 12, p.729-732, 1997.

[6] Batty, W. and Allsopp, D.W.E., IEEE Photon. Technol. Lett., 29, p.635-637, 1993.

[7] Moussa, Z., Boucaud, P., Julien, F.H., Lavon, Y., Sa'ar, A., Berger, V, Nagle, J. and Coron, N., Electron. Lett., 31, p.912-913, 1995.

[8] Zucker, J.E., Divino M.D., Chang, T.Y. and Sauer, N.J., Electron. Lett., 30, p.518-520, 1994.

[9] Khurgin, J. and Li, S., Appl. Phys. A, 53, p.523-534, 1991.

[10] Kaduki K.A., AJST, 1, p.1-8, 2000.

[11] Silver M. and O'Reilly E.P., IEEE J. Quantum Electron., 30, p.547-553, 1994.

[12] Jones G., Smith A.D., O’Reilly E.P., Silver M., Briggs A.T.R., Fice M.J., Adams A.R., Greene P.D., Scarrott K. and Vranic A, IEEE J. Quantum Electron., 34, p.822833,1998

[13] Sweeney S.J., Phillips A.F., Adams A.R., O’Reilly E.P.,
Silver M. and Thijs P.J.A., IEEE Photon. Tech. Lett., 10, p.1076-1078, 1998

[14] Sweeney S.J., Phillips A.F., Adams A.R., O’Reilly E.P., Silver M. and Thijs P.J.A., in Tech. Dig. CLEO`98, 6, p.304, 1998.

[15] Kane E.O. in Handbook on semiconductors, vol. 1, W. Paul Ed., North-Holland, Amsterdam, p.193, 1982.

[16] Kaduki K.A. and Batty W., Physica Scripta, 61, p.213229, 2000 .

[17] Cohen A.M. and Marques G.E., Phys. Rev. B, 41, p.10608, 1990.

[18] Chang C-S and Chuang S.L., IEEE J. Sel. Topics Quantum Electron., 1, p.218-229, 1995.

[19] Agrawal G.P. and Dutta N.K., Long Wavelength Semiconducor Lasers, van Nostrand Reinhold, New York, 1986.

[20] Cappasso A., Cho A.Y., Mohammed K. and Foy P., Appl. Phys. Lett., 46, p.66-666, 1985.

[21] Choy W.C.H., Li E.H., Chan M.C.Y and Weiss B.L., IEEE J. Quantum Electron., 35, p.913-921, 1999.

[22] Matthews J.W. and Blakeslee A.E., J. Cryst. Growth, 27, p.118-125, 1974.

[23] Madelung O., Ed., Landolt-Börnstein, New Series, Group III, vol. 17, Springer, Berlin, 1982.

[24] Chuang S.L., Phys. Rev. B, 43, p.9649-9661, 1991

[25] Weihofen R., Weiser G, Stark Ch. and Simes R.J, Phys. Rev. B, 51, p.4296-4305, 1996.

[26] Afromowitz M.A., Sol. State Commun., 15, p.59-63, 1974.

[27] Utaka K., Suematsu Y., Kobayashi K. and Kawanishi H., Jpn. J. Appl. Phys., 19, p.1137-1140, 1980.

[28] Jones G. and O'Reilly E.P., IEEE J. Quantum Electron., 29, p.1344-1354, 1993.

[29] Taylor R.J., Abram R.A., Burt M.G. and Smith C, Semicond. Sci. Technol., 5, p.90-104, 1990.

[30] Hausser S. Fuchs G, Hangleiter A. Streubel K. and Tsang W.T., Appl. Phys. Lett., 55, p.913-915, 1993.

[31] Gilard O., Lozes-Dupuy F., Vassilieff G., Barrau J. and Le leune P., J. Appl. Phys., 84, p.2705-2715, 1998.

[32] Polkovnikov A.S. and Zegrya G.G., Phys. Rev. B., 58, p.4039-4056, 1998. 